\title{
CINÉTICA DE DESSORÇÃO DO ÓLEO DE ANDIROBA (Carapa guianensis): EXPERIMENTOS E MODELAGEM MATEMÁTICA
}

\author{
C. C. R. BATISTA ${ }^{1}$, R. F. MOREIRA ${ }^{2}$, J. E. RODRIGUES ${ }^{3}$, R. N. CARVALHO Jr ${ }^{4}$, N. T. \\ MACHADO $^{3}$, M. E. ARAÚJO ${ }^{3}$
}

${ }^{1}$ Universidade Federal de Pará, Programa Pós-Grad. em Eng. de Recursos Naturais da Amazônia

${ }^{2}$ Universidade Federal do Pará, Programa de Pós-Graduação em Engenharia Química

${ }^{3}$ Universidade Federal do Pará, Faculdade de Engenharia Química

${ }^{4}$ Universidade Federal do Pará, Faculdade de Engenharia de Alimentos

E-mail para contato: meaaraujo@gmail.com

\begin{abstract}
RESUMO - Este trabalho teve como objetivo a determinação de dados experimentais de cinética de dessorção do óleo da andiroba (Carapa guianensis), adsorvidos em $\gamma$ aluminas ativadas termicamente a $650^{\circ} \mathrm{C}$. Os experimentos de dessorção foram realizados em uma planta piloto de extração supercrítica a 150, 200 e 250 bar e temperatura de $50^{\circ} \mathrm{C}$. Os dados de cinética de dessorção (massa acumulada de óleo versus tempo) foram correlacionados com os modelos de Tan e Liou (1989), Goto et al. (1993) e Martínez et al. (2003) a fim de determinar os parâmetros de transferência de massa. A modelagem matemática das cinéticas de dessorção foi analisada com os seguintes parâmetros: erro relativo, erro médio relativo, desvio padrão e faixa de erro. Os modelos descreveram quantitativamente todas as cinéticas de dessorção do óleo de andiroba para todas as pressões estudadas.
\end{abstract}

\section{INTRODUÇÃO}

A separação seletiva de constituintes de extratos naturais requer na maioria dos casos a aplicação de processos integrados onde o uso da tecnologia supercrítica tem apresentado destaque. $\mathrm{Na}$ literatura, encontram-se estudos que investigam a capacidade do fluido supercrítico em processos de dessorção. Muitos destes resultados tratam das características da dessorção supercrítica de sistemas em carvão ativado (Tan e Liou, 1989; Matras et al., 1993). O processo de dessorção supercrítica empregando dióxido de carbono como solvente foi aplicado principalmente aos óleos cítricos.

A adsorção de constituinte de extratos vegetais em condições ambientais e dessorção utilizando $\mathrm{CO}_{2}$ supercrítico foi estudada para o óleo de palma. Araújo et al., (2006) verificaram que a capacidade máxima de adsorção da $\gamma$-alumina ativada a $650^{\circ} \mathrm{C}$ obtida foi de $0,339 \mathrm{~g} d e$ óleo/g de adsorvente e o rendimento máximo de dessorção com $\mathrm{CO}_{2}$ supercrítico foi de 35,04\% a pressão de 250 bar e $60^{\circ} \mathrm{C}$. Azevedo et al. (2011) estudaram a adsorção de constituintes do óleo de palma bruto em $\gamma$-aluminas ativadas a $450^{\circ} \mathrm{C}$ e $650^{\circ} \mathrm{C}$. Os experimentos de dessorção com $\mathrm{CO}_{2}$ supercrítico foram realizados a pressões de 250 e 300 bar a $60^{\circ} \mathrm{C}$. Foram obtidas frações quatro vezes mais enriquecidas de carotenoides, para os experimentos com $\gamma$-alumina a $650^{\circ} \mathrm{C}$ e dessorção a 300 bar.

Cunha et al. (2012a) investigaram o processo integrado de extração/adsorção do óleo da 
polpa de buriti em coluna empacotada com $\gamma$-alumina, usando $\mathrm{CO}_{2}$ como meio supercrítico, a pressões de 150 e 200 bar e $60^{\circ} \mathrm{C}$. Um modelo de pseudo-primeira ordem foi aplicado para descrever a cinética de transferência de massa do processo adsorção. Os resultados mostraram que o modelo cinético descreveu os dados experimentais de adsorção do óleo de buriti em $\gamma$-alumina, em meio de $\mathrm{CO}_{2}$ supercrítico, para as pressões estudadas. No trabalho de Cunha et al., (2012b), os estudos foram direcionados ao processo integrado de adsorção do óleo de buriti, em coluna empacotada com $\gamma$-alumina, e dessorção, usando $\mathrm{CO}_{2}$ como meio supercrítico, objetivando avaliar a capacidade de enriquecimento em carotenos e antioxidantes do processo. Este processo integrado de adsorção/dessorção supercrítica proporcionou a obtenção de correntes de óleo de buriti enriquecidos em 2,5 vezes à concentração de carotenoides.

Muitos modelos matemáticos com a finalidade de representar o processo de extração supercrítica foram propostos, entre eles os modelos de Tan e Liou (1989), Goto et al. (1993); Sovová (1994) e Martínez et al. (2003). Estes modelos tem como fundamento a aplicação de um balanço de massa diferencial ao longo do leito fixo para descrever as curvas cinéticas. Portanto, a partir do uso de ferramentas computacionais associadas aos modelos de transferência de massa é possível formar um banco de dados destes parâmetros que descrevam o processo, para diversos sistemas de extração de diferentes matrizes vegetais, possibilitando a utilização destes parâmetros para futuros estudos de aumento de escala de produção além de servir de base para as indústrias que utilizam essas matérias-primas em seus processos produtivos (Santana, 2013).

A andiroba (Carapa guianesis) é característica da região amazônica e dos solos úmidos entre o Amapá, Pará e a Bahia. O óleo de andiroba contém ácidos graxos livres, triglicerídeos e terpenos, estes últimos são responsáveis pela sua ação bioativa. A aplicação do processo de adsorção utilizando aluminas ativadas termicamente possibilita a eliminação parcial dos ácidos graxos livres no percolado e a adsorção dos compostos terpênicos no adsorvente. O processo de adsorção em conjunto com a dessorção dos constituintes do óleo de andiroba retidos no adsorvente com $\mathrm{CO}_{2}$ supercrítico possibilita a recuperação e o enriquecimento da fração terpênica nos extratos.

Este trabalho teve como objetivo a determinação de parâmetros de transferência de massa a partir de dados experimentais de cinética de dessorção a pressões de 150,200 e 250 bar e $50^{\circ} \mathrm{C}$, do óleo da andiroba (Carapa guianensis) adsorvidos em aluminas ativadas termicamente a $650^{\circ} \mathrm{C}$, a fim de contribuir para o processo integrado de adsorção/dessorção.

\section{MATERIAIS E MÉTODOS}

O hidróxido de alumínio utilizado como matéria prima para a preparação do adsorvente foi fornecido pela Alunorte S/A (Pará-Brasil). O óleo de andiroba, obtido por prensagem mecânica, foi fornecido pela Brasmazon S/A (Pará-Brasil) e o dióxido de carbono com 99,9 \% de pureza foi fornecido pela White Martins S/A (Pará-Brasil).

\subsection{Adsorção}

Foi aplicada a metodologia descrita por Azevedo et al (2011) para o processo de ativação térmica para a produção do adsorvente ( $\gamma$-alumina). O equipamento utilizado para realizar os experimentos de adsorção foi constituído por uma coluna cilíndrica de aço inox (unidade de adsorção) com $5,5 \mathrm{~cm}$ de diâmetro e $25 \mathrm{~cm}$ de altura acoplada a uma unidade de 
percolação/filtração com $19,5 \mathrm{~cm}$ de altura e $4,5 \mathrm{~cm}$ de diâmetro. Os dois aparatos foram encamisados e o sistema todo foi mantido aquecido por um banho termostático que utiliza água como fluido refrigerante. A descrição detalhada do equipamento e do procedimento de adsorção foi apresentada por Azevedo et al. (2011).

\subsection{Dessorção Supercrítica}

Foram realizados quatro experimentos de dessorção na unidade piloto de extração supercrítica da UFPA, a pressões de 150,200 e 250 bar e a temperatura de $50^{\circ} \mathrm{C}$ com vazões de $\mathrm{CO}_{2}$ de $16,78,16,10,17,20 \mathrm{~g} / \mathrm{min}$, respectivamente,. Neste trabalho foi utilizada somente uma parte da unidade piloto conforme descrito em trabalhos anteriores (Araújo et al., 2000; Azevedo et al., 2011), o qual consiste em utilizar o último separador contendo uma célula de aço inox adaptada com $5,5 \mathrm{~cm}$ de altura e $4,4 \mathrm{~cm}$ de diâmetro. O leito fixo apresentou as seguintes características físicas: densidade real de $690 \mathrm{~kg} / \mathrm{m}^{3}$, densidade aparente $354 \mathrm{~kg} / \mathrm{m}^{3}$, porosidade do leito de 0,487 e volume do leito com $0,000076 \mathrm{~m}^{3}$. Estes resultados foram utilizados como dados de entrada para a modelagem matemática.

\subsection{Modelagem Matemática}

Os modelos de Tan e Liou (1989), Goto et al. (1993) e Martínez et al. (2003), descritos pelas equações 1 a 3 respectivamente, foram aplicados às curvas de dessorção supercrítica do óleo de andiroba. Foi utilizado um aplicativo computacional desenvolvido em Excel por Santos (2007) e validado por Santana (2013) para a determinação dos parâmetros dos modelos de transferência de massa. O aplicativo utiliza a função SOLVER, que usa o código de otimização não linear de gradiente reduzido genérico (GRG2).

$$
\begin{aligned}
& m(h=H, t)=\frac{A}{K_{d}}\left[1-\exp K_{d}\right]\left[\exp \left(-K_{d} t\right)-1\right] \\
& m=\frac{A 1[\beta / K+(1-\beta)] X_{0} \rho_{s} Q_{C O_{2}}}{\rho}\left\{\frac{\tau}{a_{1}}\left[\exp \left(a_{1} \frac{t}{\tau}\right)-1\right]+\frac{\tau}{a_{2}}\left[1-\exp \left(a_{2} \frac{t}{\tau}\right)\right]\right\}
\end{aligned}
$$

$$
m=\frac{X_{0} F_{0}}{\exp \left(b_{i} t_{m i}\right)}\left\{\frac{1+\exp \left(b_{i} t_{m i}\right)}{1+\exp \left[b_{i}\left(t_{m i}-t\right)\right]}-1\right\}
$$

Onde:

$$
\begin{aligned}
& A=Q_{\mathrm{CO}_{2}} \frac{1-\varepsilon}{\varepsilon} \cdot X_{0} \frac{\rho_{\mathrm{CO}_{2}}}{\rho_{S}} \\
& B=\frac{\varepsilon \cdot H}{u}=X_{0} \frac{\varepsilon \cdot H \cdot S \cdot \rho_{S}}{Q_{C O_{2}}} \\
& a_{1}=\frac{1}{2}\left(+b \sqrt{b^{2}-4 c}\right) \\
& a_{2}=\frac{1}{2}\left(-b \sqrt{b^{2}-4 c}\right) \\
& A 1=\frac{(1-\varepsilon) \emptyset}{[\beta+(1-\beta) K] \varepsilon\left(a_{1}-a_{2}\right)} \\
& b=\frac{\emptyset}{[\beta+(1-\beta) K]}+\frac{1}{\varepsilon}+\frac{\emptyset(1-\varepsilon)}{\varepsilon}
\end{aligned}
$$




$$
\begin{aligned}
& c=\frac{\emptyset}{[\beta+(1-\beta) K] \varepsilon} \\
& \varnothing=K_{p} a_{p} \tau \\
& K_{p}=\frac{3 K_{f}}{3+B_{i}} \\
& X_{0}=\frac{m_{\text {ext }}}{F_{0}}
\end{aligned}
$$

Neste trabalho os valores de densidade do dióxido de carbono foram calculados de acordo com a equação de estado de SPAN-WAGNER, a partir de uma planilha em Excel (Gast, 2006).

Foram utilizados os seguintes parâmetros estatísticos para análise da modelagem matemática: erro relativo $(e)$, erro médio relativo $\left(E_{m}\right)$, desvio padrão $(R)$ e faixa de erro $(S)$, representados pelas equações 14 a 17 .

$$
\begin{aligned}
& e=\frac{m_{\text {cal }}-m_{\text {exp }}}{m_{\text {ext }}} \\
& E_{m}=\frac{1}{N} \sum_{i} e \\
& R=e_{\text {max }}-e_{\text {min }} \\
& S=\left[\frac{1}{N-1} \sum_{i}\left(e-E_{m}\right)^{2}\right]^{1 / 2}
\end{aligned}
$$

\section{RESULTADOS E DISCUSSÕES}

Os experimentos de dessorção do óleo de andiroba com $\mathrm{CO}_{2}$ supercrítico apresentaram rendimentos crescentes com a pressão a temperatura de $50^{\circ} \mathrm{C}$, com valores de $14,72 \%$, $20,54 \%$ e 28,27 \% a pressões de 150, 200 e 250 bar, respectivamente.

Os valores dos parâmetros dos modelos ajustados às cinéticas de dessorção do óleo de andiroba com $\mathrm{CO}_{2}$ supercrítico estão apresentados na Tabela 1. Nas Tabelas 2 a 4 estão

\begin{tabular}{|c|c|c|c|c|c|}
\hline \multicolumn{6}{|c|}{ Parâmetros ajustáveis } \\
\hline & e Liou (1989) & Goto & (2003) & Martínez & al.(2003) \\
\hline Pressão (bar) & $K d \times 10^{-5}\left(\mathrm{~s}^{-1}\right)$ & $\boldsymbol{K}$ & $\Phi$ & $t_{\min } / 60(\mathrm{~s})$ & bx60 $\left(s^{-1}\right)$ \\
\hline 150 & 4,7413 & 0,0101 & $8,95 \times 10^{-4}$ & 0,222 & 0,0291 \\
\hline
\end{tabular}
apresentados os parâmetros estatísticos determinados a partir dos valores calculados das cinéticas de dessorção com $\mathrm{CO}_{2}$ supercrítico, empregando os parâmetros ajustáveis dos modelos de transferência de massa de Tan e Liou (1989), Goto et al. (1993) e Martínez et al. (2003). Não foi realizada neste trabalho a análise do erro paramétrico. Nas Figuras 1a 3 estão apresentadas as comparações entre as curvas calculadas pelos modelos de transferência de massa e as curvas de dessorção experimentais.

Tabela 1 - Parâmetros dos modelos de transferência de massa 


\begin{tabular}{lrrrrr}
200 & 5,5738 & 0,0101 & $9,42 \times 10^{-5}$ & 0,253 & 0,0258 \\
250 & 4,9800 & 3,2099 & 0,0551 & 0,004 & 0,0445 \\
\hline
\end{tabular}

Os resultados demonstram que os modelos de Tan e Liou (1989), Goto et al.(1993) e Martínez et al. (2003) ajustaram quantitativamente as cinéticas de dessorção, apresentando baixos erros médios relativos. De acordo com os parâmetros estatísticos o melhor ajuste foi obtido à pressão de 250 bar pelo modelo Martínez et al. (2003).

O modelo logístico de Martínez et al. (2003) deriva do modelo de Sovová (1994), que foi proposto baseado na análise do comportamento das curvas de extração supercrítica de matrizes sólidas, onde são observadas três etapas distintas do processo de transferência de massa: etapa de taxa de extração constante, etapa de difusão do soluto do interior da partícula para a sua superfície e a terceira etapa correspondente a taxa decrescente de extração. A cinética de dessorção a 250 bar (Figura 3) foi a que mais representou as três etapas, justificando portanto o melhor ajuste do modelo.

Tabela 2- Parâmetros estatísticos para o modelo Tan e Liou (1989)

\begin{tabular}{cccc}
\hline & \multicolumn{3}{c}{ Parâmetros estatísticos } \\
\cline { 2 - 4 } Pressão (bar) & Erro médio & Faixa de erro & Desvio padrão \\
\hline 150 & 0,2996 & 2,5494 & 0,8405 \\
200 & 0,0470 & 0,4509 & 0,1346 \\
250 & 0,1208 & 0,5020 & 0,2310 \\
\hline
\end{tabular}

Tabela 3- Parâmetros estatísticos para o modelo Goto et al. (1993)

\begin{tabular}{cccc}
\hline & \multicolumn{3}{c}{ Parâmetros estatísticos } \\
\cline { 2 - 4 } Pressão (bar) & Erro médio & Faixa de erro & Desvio padrão \\
\hline 150 & 0,4069 & 3,2199 & 1,0484 \\
200 & 0,0268 & 0,2569 & 0,0895 \\
250 & 0,0714 & 0,3348 & 0,1496 \\
\hline
\end{tabular}

Tabela 4- Parâmetros estatísticos para o modelo Martínez et al. (2003)

\begin{tabular}{cccc}
\hline & \multicolumn{3}{c}{ Parâmetros estatísticos } \\
\cline { 2 - 4 } Pressão (bar) & Erro médio & Faixa de erro & Desvio padrão \\
\hline 150 & 0,2020 & 1,8976 & 0,6148 \\
200 & 0,0845 & 0,6807 & 0,1977 \\
250 & 0,0027 & 0,1561 & 0,0559 \\
\hline
\end{tabular}




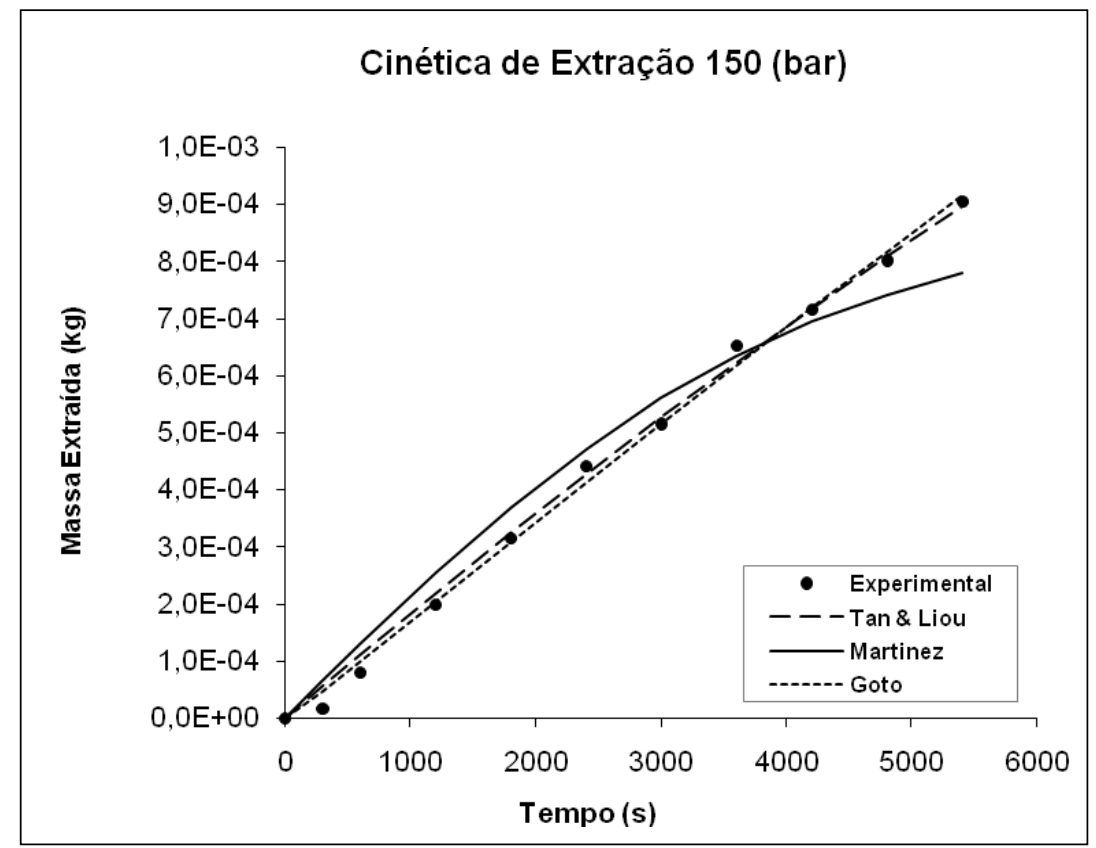

Figura 1. Modelagem da cinética de dessorção do óleo da andiroba a 150 bar.

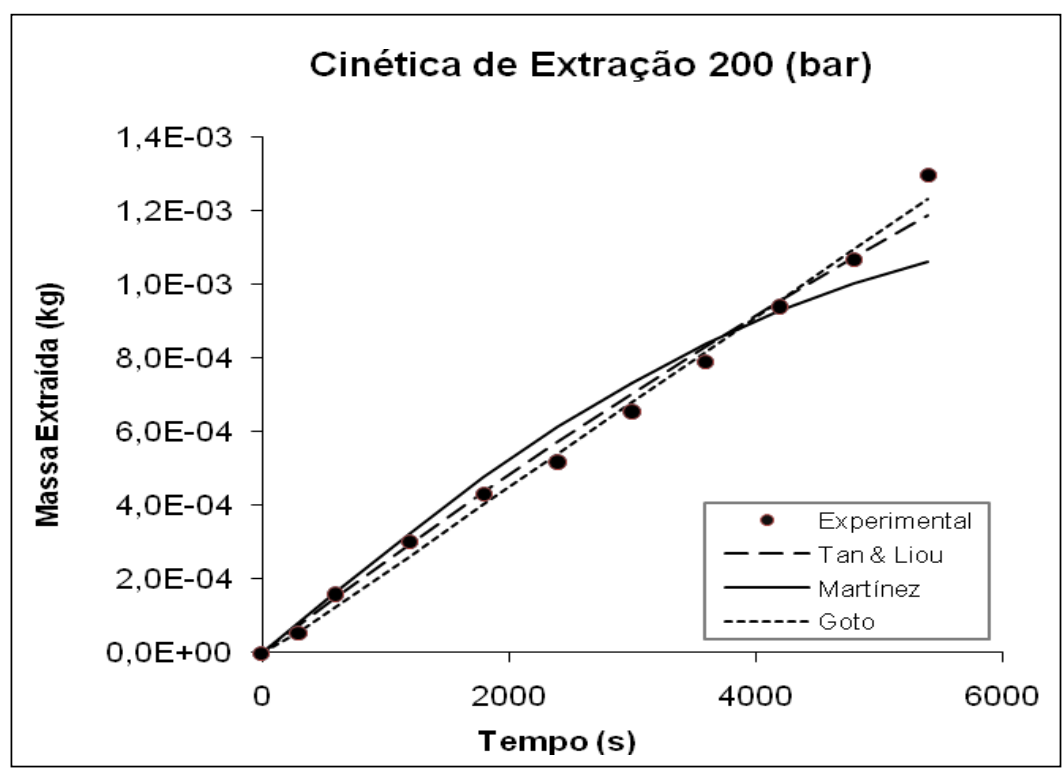

Figura 2. Modelagem da cinética de dessorção do óleo da andiroba a 200 bar. 


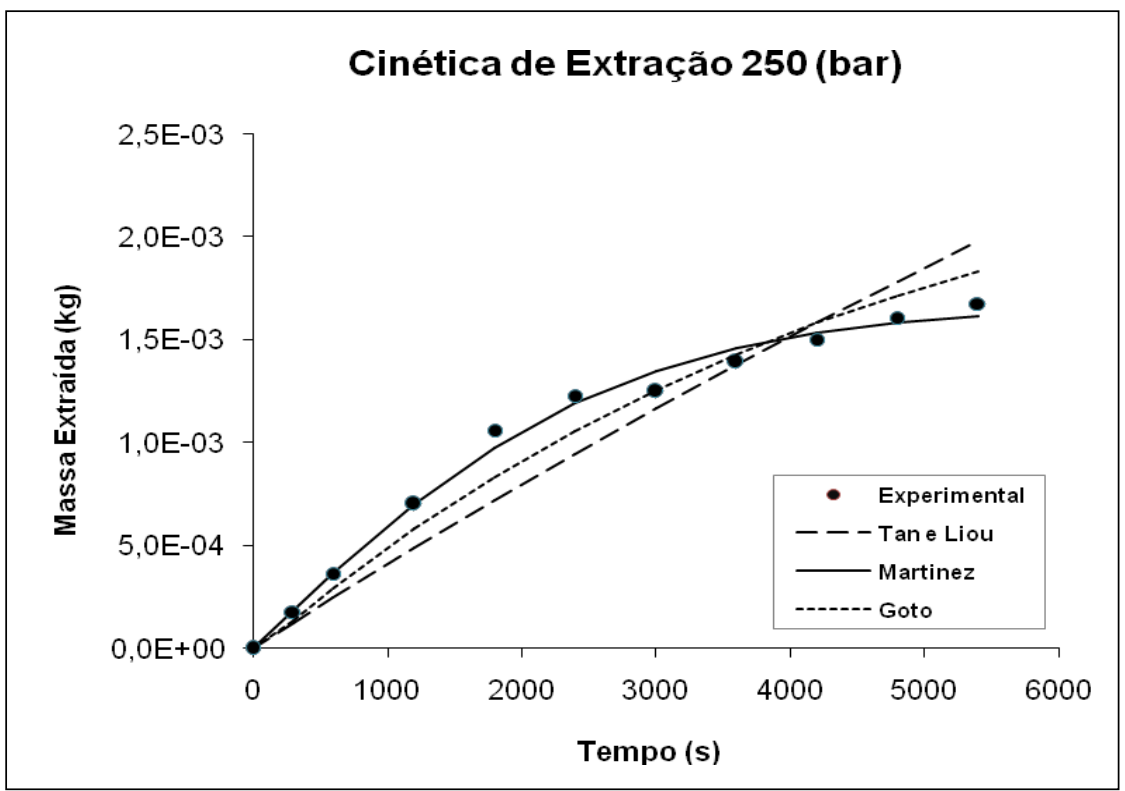

Figura 3. Modelagem da cinética de dessorção do óleo da andiroba a 250 bar.

\section{CONCLUSÕES}

Este trabalho teve como objetivo geral avaliar o processo de dessorção com dióxido de carbono supercrítico do óleo de andiroba adsorvidos em $\gamma$-alumina ativada termicamente a $650^{\circ} \mathrm{C}$. Foram medidos dados experimentais de cinética de dessorção, a pressões de 150, 200 e 250 bar a temperaturas de $50^{\circ} \mathrm{C}$. Verificou-se que o rendimento global da dessorção aumenta com o aumento da pressão.

Os dados de cinética de dessorção (massa acumulada de óleo versus tempo) foram correlacionados com os modelos de Tan e Liou (1989), Goto et al. (1993) e Martínez et al. (2003) a fim de determinar os parâmetros de transferência de massa. Os modelos descreveram quantitativamente todas as cinéticas de dessorção do óleo de andiroba para todas as pressões estudadas.

\section{NOMENCLATURA}

$\tau=$ tempo de residência do solvente no leito (s);

$K_{f}=$ Coeficiente volumétrico de transferência de massa $\left(\mathrm{m}^{-3}\right)$, para o modelo Goto et al. (1993);

$a_{p}=$ Área específica de transferência de massa (m);

$B_{i}=$ número de Biot;

$K_{p}=$ transferência de massa combinado, referente ao modelo Goto et al. (1993);

$K=$ constante absortiva de equilíbrio, referente ao modelo Goto et al. (1993);

$\varnothing=$ coeficiente global de transferência de massa, referente ao modelo Goto et al. (1993);

$b_{\mathrm{i}}=$ parâmetro ajustável $\left(\mathrm{s}^{-1}\right)$, referente ao modelo de Martínez et al. (2003); 
$F_{0}=$ massa da alimentação $(\mathrm{Kg})$;

$K_{d}=$ coeficiente de dessorção $\left(\mathrm{s}^{-1}\right)$;

$S=$ área $\left(\mathrm{m}^{2}\right)$;

$t=$ tempo $(\mathrm{s})$;

$t_{m i}=$ parâmetro ajustável (s), referente ao modelo de Martínez et al. (2003);

$m_{\text {ext }}=$ massa extraída;

$X_{0}=$ rendimento global

\section{REFERÊNCIAS}

ARAÚJO, M. E.; AMARAL, A. R.; RODRIGUES, J. E.; SANTOS, J. L.; TREJO, A. A. M.; MACHADO, N. T.; Solubilidade de óleo de palma e modelagem da dessorção com dióxido de carbono supercrítico. Anais do XVI Congresso Brasileiro de Engenharia Química, CD-ROM, Santos, SP, Brasil, 2006.

ARAÚJO, M.E.; MACHADO, N.T.; FRANÇA, L.F.; MEIRELES, M.A.A. Supercritical Extraction of Pupunha (Guilielma speciosa) Oil in a Fixed bed Using Carbon Dioxide. Brazilian Journal of Chemical Engineering, v.17, n.3, p.297-306, 2000.

AZEVEDO, F.F.M.; CUNHA, M.A.E.; NEVES, R.F.; FRANÇA, L.F.; MACHADO, N.T. Enriching of Carotenes from Palm Oil by Adsorption and Supercritical $\mathrm{CO}_{2}$ Desorption. The International Review of Chemical Engineering, v.3, p. 359-369, 2011.

CUNHA, M.A.E.; SANTANA, A.L.; FRANÇA, L.F.; ARAUJO, M.E.; MACHADO, N.T. Tecnologia Supercrítica Aplicada - Adsorção de Constituintes de Óleo Vegetal em Aluminas Ativada. . Anais do XVI Congresso Brasileiro de Engenharia Química, CDROM, Búzios, RJ, Brasil, 2012a.

CUNHA, M. A. E.; NEVES, R. F.; SOUZA, J. N. S.; FRANCA, L. F.; ARAUJO, M. E.; BRUNNER, G.; MACHADO, N. T. Supercritical adsorption in $\gamma$-alumina: A methodology of buriti oil (Mauritia flexuosa, Mart.) for the enriching of anti-oxidants. The Journal of Supercritical Fluids, v. 66, p. 181-191, $2012 \mathrm{~b}$.

GAST, K. Enrichment of Vitamin E and Provitamin A from Palm Oil Derivates with Supercritical Fluids. Berichte aus der Verfahrenstechnik. TUHH. Shaker Verlag. Aachen, 2006.

GOTO, M.; SATO, M.; HIROSE, T. Extraction of peppermint oil by supercritical carbon dioxide. J. Chem. Eng. Japan, n. 26, p. 401 - 407, 1993.

MADRAS, G.; ERKEY, C.; AKGERMAN, A. Supercritical Fluid Regeneration of Activated Carbon Loaded with Heavy Molecular Weight Organics. Industrial and Engineering Chemistry Research v. 32, p. 1163-1168, 1993.

MARTÍNEZ, J.; MONTEIRO, A. R.; ROSA, P. T. V.; MARQUES, M. O. M.; MEIRELES, M. A. A. Multicomponent Ginger Oleoresin with Supercritical Carbon Dioxide. Industrial and Engineering of Chemistry Research. V. 42, p. 1057-1063, 2003.

TAN, C.; LIOU, D. Modeling of Desorption at. American Institute of Chemical Engineers Journal, v. 6, n. 35, p. $1029-1031,1989$. 
SANTANA, A L. Determinação de Parâmetros de Transferência de Massa do Processo de Extração Supercrítica. Dissertação - Universidade Federal do Pará, Programa de PósGraduação em Engenharia Química, Belém, 2013.

SANTOS, J.L. Projeto de uma Unidade de Extração Supercrítica Empregando Simulador de Processos. Dissertação - Universidade Federal do Pará, Programa de Pós-Graduação em Engenharia Química, Belém, 2007.

SOVOVÁ, H. Rate of the Vegetable Oil Extraction with Supercritical CO2: I. Modeling of Extraction Curves. Chem. Eng. Science, v. 3, n. 49, p. 409 - 414, 1994. 